\title{
Honokiol Ameliorates Amyloidosis and Neuroinflammation and Improves Cognitive Impairment in Alzheimer's Disease Transgenic Mice
}

\author{
Dongmei Wang, Xiaohui Dong, and Chenying Wang
}

Department of Pathogen Biology, Medical College, Henan University of Science and Technology, Luolong District, Luoyang, China

Received February 24, 2018; accepted July 5, 2018

\section{ABSTRACT}

The present study examined the effects of honokiol on amyloid- $\beta$ $(A \beta)$-induced cognitive impairment and the underlying mechanisms in APPswe/PS1dE9 transgenic mice. The results showed that honokiol administration ( $20 \mathrm{mg} / \mathrm{kg}$ per day, intraperitoneally) for 6 weeks effectively improved spatial memory deficits in APPswe/PS1dE9 transgenic mice. Honokiol significantly lowered $\mathrm{A} \beta$ production and senile plaque deposition by downregulating $\beta$-site amyloid precursor protein cleavage enzyme 1 and enhancing $A \beta$ phagocytosis by microglia. Honokiol reduced glial cell activation and the production of proinflammatory cytokines
(TNF- $\alpha$, IL-1 $\beta$, and IL-6). Honokiol increased the transcriptional activity and protein levels of peroxisome proliferatoractivated receptor- $\gamma$ (PPAR $\gamma$ ). However, all of the beneficial effects of honokiol on pathologic changes, including biochemistry and cognitive function, could be blocked by GW9662, a specific PPAR $\gamma$ inhibitor. These findings suggested that honokiol may be a natural PPAR $\gamma$ agonist, acting to attenuate $\mathrm{A} \beta$ generation and neuroinflammation. Therefore, honokiol may be a potential therapeutic approach for Alzheimer's disease.

\section{Introduction}

Alzheimer's disease $(\mathrm{AD})$ is the most common neurodegenerative disease and it is characterized by progressive cognitive dysfunction. Extracellular senile plaques, intracellular neurofibrillary tangles, and massive neuronal loss in the brain are prominent neuropathological hallmarks of $\mathrm{AD}$ (Hardy and Selkoe, 2002; Steel, 2010). Although the exact mechanism of $\mathrm{AD}$ pathogenesis remains unknown, extensive studies suggest that the accumulation and aggregation of amyloid- $\beta(\mathrm{A} \beta)$ in brain areas is associated with synaptic loss, neuronal death, and cognitive decline (Selkoe and Hardy, 2016).

Clinical and epidemiologic studies indicate a close link between $\mathrm{AD}$ and diabetes mellitus. Patients among those with type 2 diabetes mellitus have a higher risk of $\mathrm{AD}$ (Maher and Schubert, 2009), and $\mathrm{AD}$ may be considered to be type 3 diabetes (de la Monte and Wands, 2008) given the evidence for central insulin resistance as well as dysregulated glucose metabolism observed in the brains of $\mathrm{AD}$ patients (Talbot et al., 2012). Thiazolidinediones (TZDs) are an important class of insulin sensitizers used in the treatment of type 2 diabetes mellitus. These drugs are known to act by increasing the transactivation activity of peroxisome proliferator-activated

The authors declare that they have no conflict of interest.

The present work was supported by the National Natural Science Foundation of China [Grant 81601225] and the Young Backbone Teachers Assistance Scheme of Henan Province Colleges and Universities.

https://doi.org/10.1124/jpet.118.248674. receptor- $\gamma(\operatorname{PPAR} \gamma)$. Recent studies have shown TZDs to be a potential treatment of AD (Escribano et al., 2010; Pérez and Quintanilla, 2015). TZDs downregulate $\beta$-site amyloid precursor protein cleavage enzyme 1 (BACE1) and reduce $\mathrm{A} \beta$ generation (Searcy et al., 2012). It has been reported that PPAR $\gamma$ agonists can suppress $\mathrm{A} \beta$-induced glial activation and neuroinflammation, enhance microglia activation into antiinflammatory phenotypes, and facilitate $\mathrm{A} \beta$ phagocytosis by microglia (Mandrekar-Colucci et al., 2012). Taken together, this growing body of research indicates that PPAR $\gamma$ agonists may represent new drugs for the treatment of $\mathrm{AD}$. However, current PPAR $\gamma$-targeting drugs, such as TZD treatment, have been associated with undesirable side effects; therefore, it is urgent to seek an alternative drug for $\mathrm{AD}$ therapy that possesses the capacity for PPAR $\gamma$ activation with minimal side effects.

Honokiol, isolated from traditional Chinese herbal drug magnolia bark, exhibits broad pharmacological functions, such as anti-inflammatory, antithrombotic, antidepressant, analgesic, and antihypertrophic effects. Honokiol has been shown to have good central nervous system penetration (Wang et al., 2011) and potential effects in the prevention and treatment of neurodegenerative disorders (Wang et al., 2014). Oral administration of honokiol attenuates age-related cognitive impairment and neuronal injury in senescence-accelerated mice (Matsui et al., 2009). Honokiol improves scopolamine-induced cognitive deficits in mice. Moreover, honokiol can ameliorate lipopolysaccharide-induced memory deficits via its

ABBREVIATIONS: AD, Alzheimer's disease; $\mathrm{A} \beta$, amyloid- $\beta$; BACE1, $\beta$-site amyloid precursor protein cleavage enzyme 1 ; ELISA, enzyme-linked immunosorbent assay; PPAR $\gamma$, peroxisome proliferator-activated receptor- $\gamma$; TZD, thiazolidinedione; WT, wild type. 
antiamyloidogenic and antineuroinflammatory effects (Lee et al., 2013). Research has indicated that honokiol acts as a PPAR $\gamma$ agonist via directly binding to the purified PPAR $\gamma$ ligand-binding domain without the side effects of pioglitazone (Atanasov et al., 2013). Whether long-term treatment with honokiol could ameliorate cognitive impairment and whether the activation of PPAR $\gamma$ by honokiol is responsible for its neuroprotective effect in $\mathrm{AD}$ remain unclear. In this study, the effect of honokiol consumption on the improvement of cognitive decline, $\mathrm{A} \beta$ burden, neuroinflammation, and PPAR $\gamma$ activation was analyzed in $\mathrm{AD}$ transgenic mice.

\section{Materials and Methods}

Animals and Treatment. Six-month-old male APPswe/PS1dE9 transgenic mice and wild-type (WT) littermates were used $(n=15)$. The generation of mice expressing human mutated forms, APPswe and PS1dE9, has already been described (Yuan et al., 2011). Mice received a daily intraperitoneal injection of GW9662 $(4 \mathrm{mg} / \mathrm{kg})$, a special PPAR $\gamma$ inhibitor, or its solvent (dimethylsulfoxide) 2 hours prior to the daily intraperitoneal administration of honokiol (20 $\mathrm{mg} / \mathrm{kg}$ ) or its solvent (dimethylsulfoxide). Treatments were administered to the following groups of mice for 6 weeks: group 1 (WT), WT littermates received a daily injection of honokiol solvent and GW9662 solvent; group 2 (APPswe/PS1dE9), APPswe/PS1dE9 mice received a daily injection of honokiol solvent and GW9662 solvent; group 3 (APPswe/PS1dE9 + honokiol), APPswe/PS1dE9 mice received a daily injection of honokiol and GW9662 solvent; group 4 (APPswe/PS1dE9 + honokiol + GW9662), APPswe/PS1dE9 mice received a daily injection of honokiol and GW9662; group 5 (GW9662), WT littermates received a daily injection of honokiol solvent and GW9662; and group 6 (honokiol), WT littermates received a daily injection of honokiol and GW9662 solvent. Honokiol (purity $>98 \%$ by high-performance liquid chromatography analysis) and PPAR $\gamma$ inhibitor GW9662 were dissolved in 10\% dimethylsulfoxide. All experimental protocols and animal usage were approved by the Institutional Animal Experiment Committee of Henan University of Science and Technology, Luoyang, China.

Morris Water Maze. The Morris water maze consisted of a circular white tank (100 $\mathrm{cm}$ in diameter) filled with nontoxic opaque water $\left(22 \pm 1^{\circ} \mathrm{C}\right)$. A removable platform $(10 \mathrm{~cm}$ in diameter $)$ was submerged $0.5 \mathrm{~cm}$ beneath the surface of the water at a constant location in the center of the target quadrants. During the learning phase, each mouse was experimented to four trials per day for five consecutive days. The time to find the platform (escape latency) was recorded and calculated as the average of four trials. A probe trial was

A
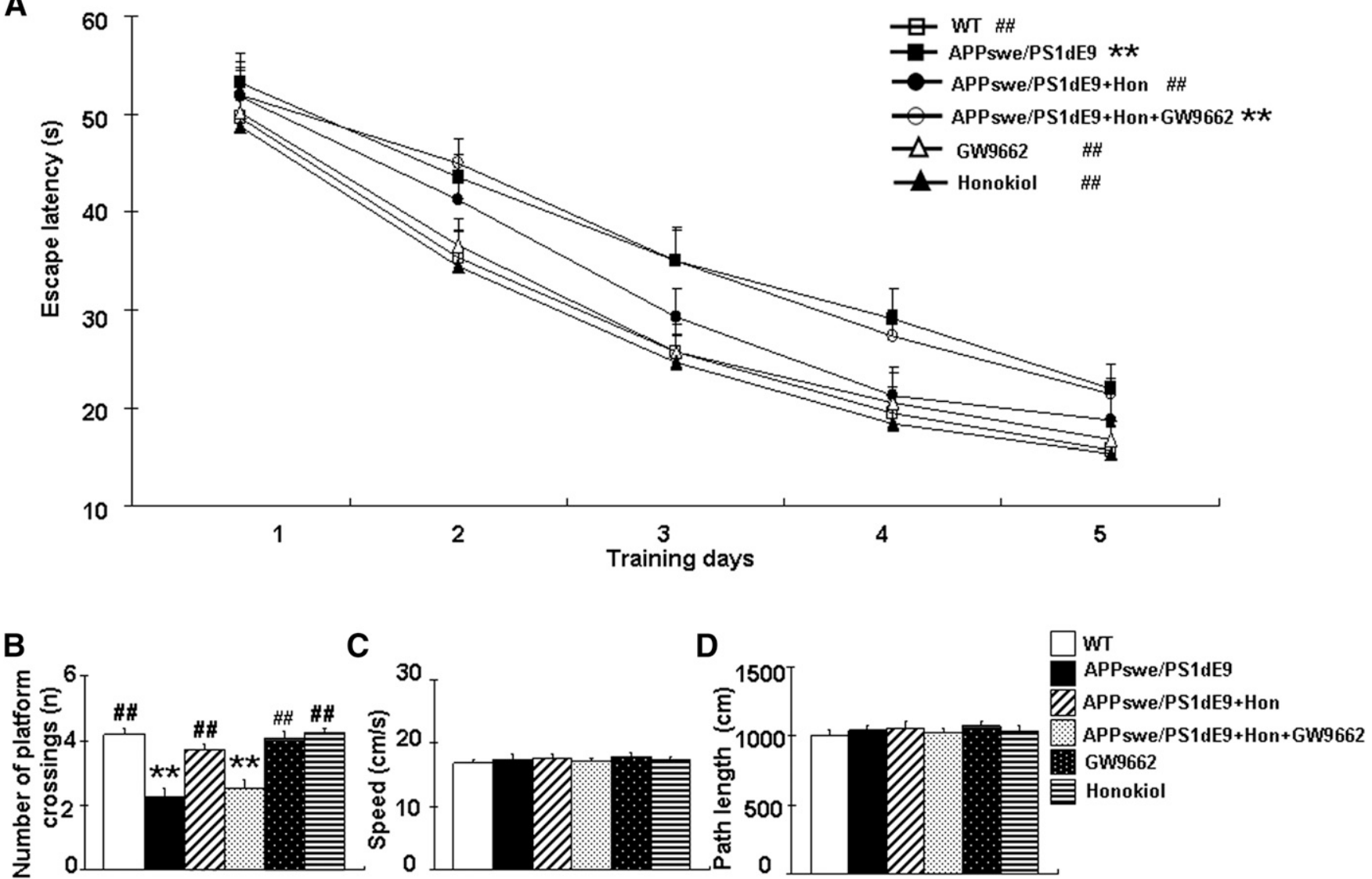

Fig. 1. Honokiol (Hon) ameliorated spatial learning and memory impairment in APPswe/PS1dE9 transgenic mice as tested using the Morris water maze. Nontransgenic littermates and APPswe/PS1dE9 mice received a daily intraperitoneal injection of GW9662 or its solvent [dimethylsulfoxide (DMSO)] 2 hours prior to daily intraperitoneal administration of honokiol or its solvent (DMSO) for 6 weeks. After treatment, WT mice, APPswe/PS1dE9 mice, APPswe/PS1dE9 + Hon mice, APPswe/PS1dE9 + Hon + GW9662 mice, GW9662 mice, and Honokiol mice underwent the Morris water maze test. (A) The escape latency during 5 days of hidden platform tests. APPswe/PS1dE9 + Hon mice showed shorter escape latencies compared with APPswe/PS1dE9 mice. (B) The number of platform crossings. The crossings were significantly increased in APPswe/PS1dE9 + Hon mice compared with APPswe/PS1dE9 mice. However, the positive effects of honokiol on cognition were blocked by coadministration of GW9662. (C) The swimming speed. (D) The path length in the probe test. All of the data are presented as mean \pm S.E.M. $(n=15$ per group) and were analyzed by two-way analysis of variance on ranks for repeated measures, followed by a Bonferroni post-hoc test for multiple comparisons. $* * P<0.01$ vs. WT mice; ${ }^{\#} P<0.01$ vs. APPswe/PS1dE9 mice. 

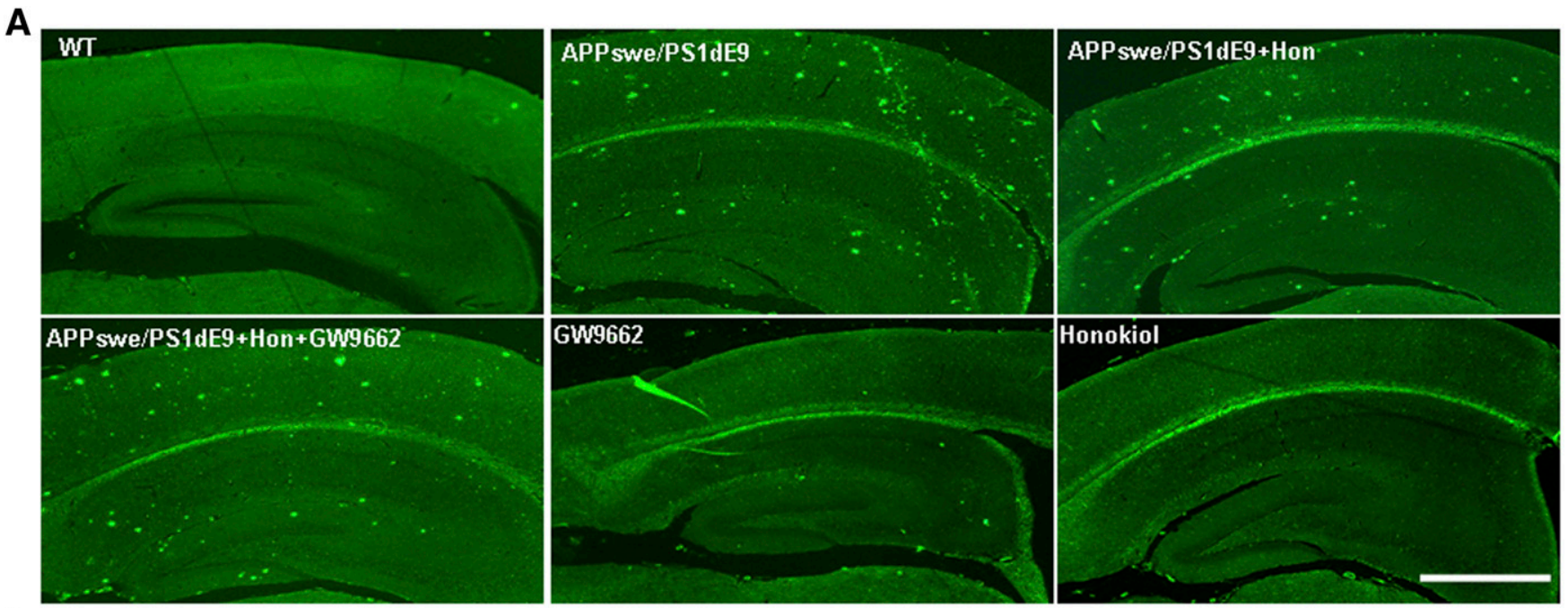

B
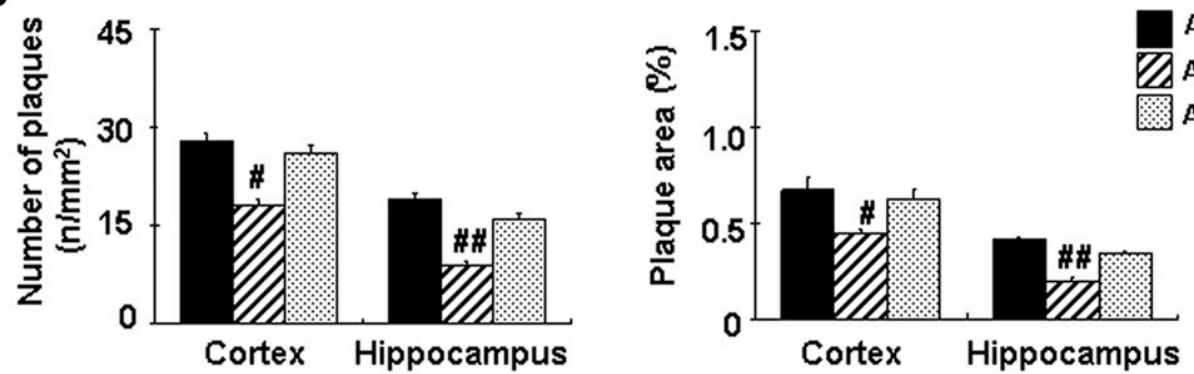

APPswe/PS1dE9 APPswe/PS1dE9+Hon APPswe/PS1dE9+Hon+GW9662

Fig. 2. Honokiol (Hon) treatment reduced A $\beta$ deposition in APPswe/PS1dE9 transgenic mice. Brain tissues from all of the mice were used in standard pathologic procedures, and sections were stained with Thioflavin-S to visualize the deposition of A $\beta$. (A) Representative brain sections showing that honokiol treatment significantly decreased Thioflavin-S immunoreactivity in the brains of APPswe/PS1dE9 mice (scale bar, $1 \mathrm{~cm}$ ). However, the inhibitory effects of honokiol on A $\beta$ deposition were blocked by coadministration of GW9662. (B) Graphs showing plaque count and the percentage of area occupied by plaques in the cortex and hippocampus. All of the data are presented as mean \pm S.E.M. $(n=4$ per group) and were analyzed by one-way analysis of variance followed by a Bonferroni post-hoc test for multiple comparisons. ${ }^{\#} P<0.05 ;{ }^{\# \#} P<0.01$ vs. APPswe/PS1dE9 mice.

carried out in the sixth day to evaluate spatial memory retention. Each mouse was placed in the water tank without the platform and allowed to swim freely for 60 seconds and the platform crossings were recorded. Performance was tracked by the EthoVision video tracking system (Version XT; Noldus, Wageningen, Netherlands).

Histologic Analysis. Paraformaldehyde-fixed brains were sectioned $(30 \mu \mathrm{m})$ coronally by using a microtome (Leica, Nussloch, Germany) and stored at $4^{\circ} \mathrm{C}$ in phoaphate buffered solution. For Thioflavine-S staining, the brain slices were incubated in a solution of $0.015 \%$ Thioflavin-S for 3 minutes at room temperature. Finally, the slices were washed in $50 \%$ ethanol and coverslipped with Permount.

Immunohistochemistry of anti-GFAP antibody (1:1000; Proteintech), Rosemont detecting activated astrocytes and anti-Iba1 antibody (1:2000; Abcam, Cambridge, UK) detecting activated microglia were used to visualize astrocytosis and microgliosis. The brain sections were incubated with the horseradish peroxidase-labeled secondary antibodies for 60 minutes and visualized by 3,3'-Diaminobenzidine (DAB). The stained sections were observed under light microscopy, and the intensity of the stained area of each group was evaluated quantitatively using Image-ProPlus version 6.0 (Media Cybernetics, Silver Spring, MD).

Double immunofluorescence staining of Iba1 and $\mathrm{A} \beta$ was performed to evaluate the phagocytic ability of microglia. The brain sections were incubated with the following primary antibodies: Iba1 (1:100; Abcam) and A $\beta$ (1: 200; Cell Signaling Technology, Boston, MA) at $4^{\circ} \mathrm{C}$ overnight. After three washes with phosphate-buffered saline, antirabbit or mouse secondary antibody conjugated to Alexa Fluor 488 or 594 (Cell Signaling Technology) was added at $37^{\circ} \mathrm{C}$ for 30 minutes.
All of the fluorescence staining images were captured on a Fluoview FV10i confocal microscope (Olympus, Tokyo, Japan). The captured images were viewed and analyzed with MetaMorph Image Analysis Software (Molecular Devices, Union City, CA).

Enzyme-Linked Immunosorbent Assay Test for A $\beta 1-40$ and A $\beta 1-42$. Soluble and insoluble $A \beta 1-40$ and $A \beta 1-42$ of the cortex and hippocampus were extracted according to a previous study (Handattu et al., 2009). In brief, the frozen mouse cortex and hippocampus were homogenized in ice-cold $20 \mathrm{mM}$ Tris, $\mathrm{pH} 8.5$ (soluble) or $5 \mathrm{M}$ guanidine $\mathrm{HCl} / 50 \mathrm{mM}$ Tris-HCl, $\mathrm{pH} 8.0$ (insoluble). The content of $\mathrm{A} \beta 1-40$ and A $\beta 1-42$ levels was determined by enzyme-linked immunosorbent assay (ELISA) kits (Invitrogen, Carlsbad, CA).

Estimation of Proinflammtory Cytokine (TNF- $\alpha$, IL-1 $\beta$, and IL-6) Levels. After mouse brain tissue was homogenized in lysis buffer and centrifuged, the supernatants were collected and sampled in triplicate to detect the levels of TNF- $\alpha$, IL- $1 \beta$, and IL- 6 by an ELISA kit (R\&D Systems, Minneapolis, MN, and Invitrogen) according to the manufacturer's instructions.

PPAR $\gamma$ Transcriptional Activity Assay. PPAR $\gamma$ transcription factor activity was measured by using an ELISA-based kit (Cayman Chemicals, Ann Arbor, MI). Nuclear extracts were incubated in a 96-well plate coated with immobilized oligonucleotides containing peroxisome proliferator responsive element. PPAR $\gamma$ contained in nuclear extract was detected by using primary antibody specific for PPAR $\gamma$ followed by a horseradish peroxidase-conjugated secondary antibody and colorimetric readout at $450 \mathrm{~nm}$.

BACE1 Enzymatic Activity Assays. BACE1-specific enzymatic activity was performed using a fluorometric reaction kit (R\&D Systems). Briefly, brain tissues were lysed with the provided extraction buffer and 
A

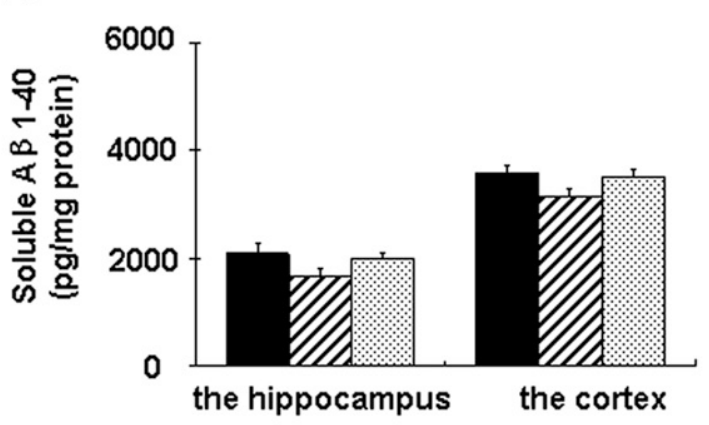

B

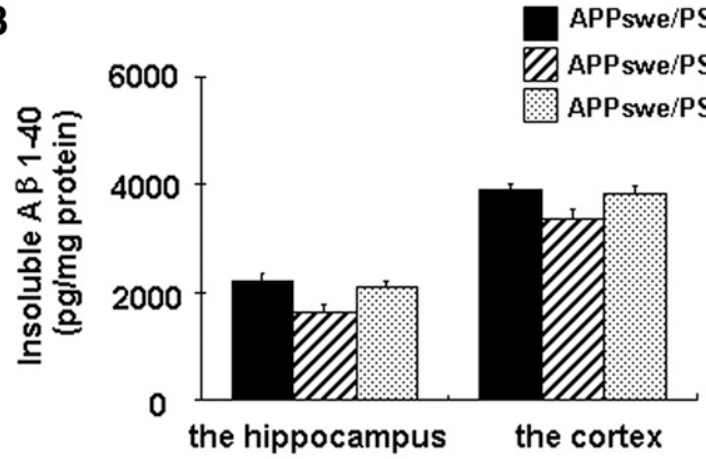

C

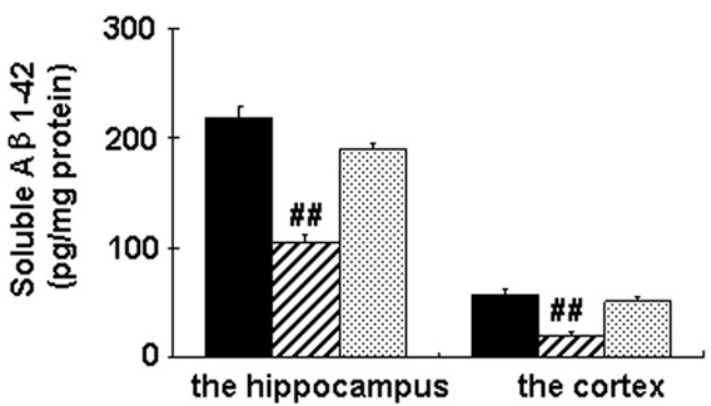



Fig. 3. Honokiol (Hon) treatment decreased A $\beta 1-42$ levels in APPswe/PS1dE9 transgenic mice. Soluble and insoluble A $\beta 1-40$ and A $\beta 1-42$ from the APPswe/PS1dE9 mice, APPswe/PS1dE9 + Hon mice and APPswe/PS1dE9 + Hon + GW9662 mice were extracted and measured by ELISA. (A) The soluble A $\beta 1-40$ levels. (B) The insoluble A $\beta 1-40$ levels. No significant difference was observed in soluble A $\beta 1-40$ levels or insoluble A $\beta 1-40$ levels among the three groups. (C) The soluble A $\beta 1-42$ levels. (D) The insoluble A $\beta 1-42$ levels in the hippocampus and cortex. Honokiol treatment significantly reduced A $\beta 1-42$ levels in the brains of APPswe/PS1dE9 mice. However, the inhibitory effects of honokiol on A $\beta 1-42$ levels were blocked by coadministration of GW9662. All of the data are presented as mean \pm S.E.M. ( $n=4$ per group) and were analyzed by one-way analysis of variance followed by a Bonferroni post-hoc test for multiple comparisons. ${ }^{\# \#} P<0.01$ vs. APPswe/PS1dE9 mice.

centrifuged at $25,000 \mathrm{~g}$ for 30 minutes. The supernatant was then incubated with reaction buffer containing $\beta$-secretase substrate. Fluorescence intensity was measured with a microplate reader at 320 and $420 \mathrm{~nm}$ as excitation and emission wavelengths, respectively.

Western Blot Analysis. Protein isolation and immunoblotting procedures were performed as previously described (Li et al., 2013; Liu et al., 2013). Primary antibodies for immunoblotting included antiGFAP (1:1000; Proteintech), anti-Iba1 (1:500; Abcam), anti-PPAR $\gamma$ (1:500; Abcam), and anti-BACE1 (1:1000; Abcam). Samples were separated by SDS-PAGE, transferred to a nitrocellulose membrane, and probed with the stated antibodies overnight at $4^{\circ} \mathrm{C}$. The membrane was then incubated with horseradish peroxidase-conjugated secondary antibodies. Immunoblots were visualized using a chemiluminescent detection system.

Statistical Analysis. All data are presented as the mean \pm S.E.M. Data were examined by one-way analysis of variance (ANOVA) followed by a Bonferroni post-hoc test for multiple comparisons using SPSS 13.0 (IBM SPSS, Chicago, IL). For the Morris water maze test, the group differences of escape latencies were analyzed using two-way analysis of variance with repeated measures. Significant differences were determined at $P<0.05$.

\section{Results}

Honokiol Alleviates Spatial Learning and Memory Deficits in APPswe/PS1dE9 Mice. To evaluate the effects of honokiol on spatial reference learning and memory impairments, we tested spatial learning and memory by the Morris water maze test. In the place navigation test, the APPswe/PS1dE9 mice showed longer escape latencies compared with the WT group $(P<0.01$ for days $2-5)$. Administration of honokiol significantly decreased the escape latencies $(P<0.05$ for days 2 to $3 ; P<0.01$ for days 4 to 5 ).

In the subsequent probe test, the number of platform crossings decreased by $47.6 \%$ in APPswe/PS1dE9 mice compared with WT mice $(P<0.01)$, indicative of cognitive impairment. Compared with APPswe/PS1dE9 mice, the number of platform crossings of the APPswe/PS1dE9 + honokiol mice was significantly increased by $64.3 \%(P<0.01)$. However, the positive effects of honokiol on the cognition were blocked by coadministration of GW9662, an inhibitor of $\operatorname{PPAR} \gamma$. Intraperitoneal injection of GW9662 into the WT group did not influence memory function. Mice treated with only honokiol showed no significant differences in the number of platform crossings in comparison with WT mice $(P>0.05)$. In addition, no significant difference in swimming speed (Fig. 1C) or path length (Fig. 1D) was found in the probe test between the six groups of mice $(P>0.05)$.

Honokiol Treatment Reduces A $\beta$ Levels and Deposition in APPswe/PS1dE9 Mice. To investigate whether honokiol treatment would inhibit $\mathrm{A} \beta$ deposition in the cortex and hippocampus, sections of mouse brains from different groups were stained with Thioavine-S, which specifically binds amyloid plaques. As shown in Fig. 2, the WT mice had no visible $\mathrm{A} \beta$ plaque. However, Thioflavin-S-positive dense-core plaques accumulated in the hippocampus and cortex of the APPswe/PS1dE9 transgenic mice, whereas the APPswe/PS1dE9 mice treated with honokiol exhibited $52.6 \%$ and $35.7 \%$ fewer Thioavine-S positive compact plaques and $51.2 \%$ and $34.1 \%$ less plaque area in hippocampus $(P<0.01)$ and cortex $(P<0.05)$, 




Fig. 4. Honokiol (Hon) suppressed neuroinflammation in APPswe/PS1dE9 transgenic mice. (A) The levels of TNF, IL-1 $\beta$, and IL-6 in mouse brain homogenates of all of the groups ( $n=3$ per group). Honokiol treatment significantly lowered the levels of TNF, IL- $1 \beta$, and IL-6 in the brains of APPswe/PS1dE9 mice. (B) Representative photomicrographs of immunohistochemistry for Iba1 in the hippocampal CA1 region of all of the groups (scale bar, $100 \mu \mathrm{m})$, and quantitative analysis of Iba1 staining intensity $(n=4$ per group). Insets indicate higher magnification of Iba1-positive cells from the CA1 area. APPswe/PS1dE9 + Hon mice exhibited a smaller number of Iba1 positive cells than APPswe/PS1dE9 mice. (C) Representative photomicrographs of immunohistochemistry for GFAP in the hippocampal CA1 region of all of the groups (scale bar, $100 \mu \mathrm{m}$ ), and quantitative analysis of GFAP staining intensity ( $n=4$ per group). Insets indicate higher magnification of GFAP positive cells from the CA1 area. APPswe/PS1dE9 + Hon mice showed a smaller number of GFAP-positive cells than APPswe/PS1dE9 mice. (D) Western blot of Iba1 and GFAP in the hippocampus $(n=3$ per group). The expressions of Iba1 and GFAP were decreased in APPswe/PS1dE9 + Hon mice compared with APPswe/PS1dE9 mice. However, the inhibitory effects of honokiol on neuroinflammation were blocked by coadministration of GW9662. All of the data are presented as mean \pm S.E.M. and were analyzed by oneway analysis of variance followed by a Bonferroni post-hoc test for multiple comparisons. ${ }^{* *} P<0.01$ vs. WT mice; ${ }^{\# \#} P<0.01$ vs. APPswe/PS1dE9 mice.

respectively, when compared with APPswe/PS1dE9 transgenic mice. Furthermore, we measured the levels of soluble $\mathrm{A} \beta$ peptide fraction and insoluble $\mathrm{A} \beta$ peptide fraction in mouse brains using ELISA. Compared with APPswe/PS1dE9 mice, treatment with honokiol significantly decreased levels of soluble $\mathrm{A} \beta 1-42$ by approximately $52.3 \%$ in the hippocampus 
A

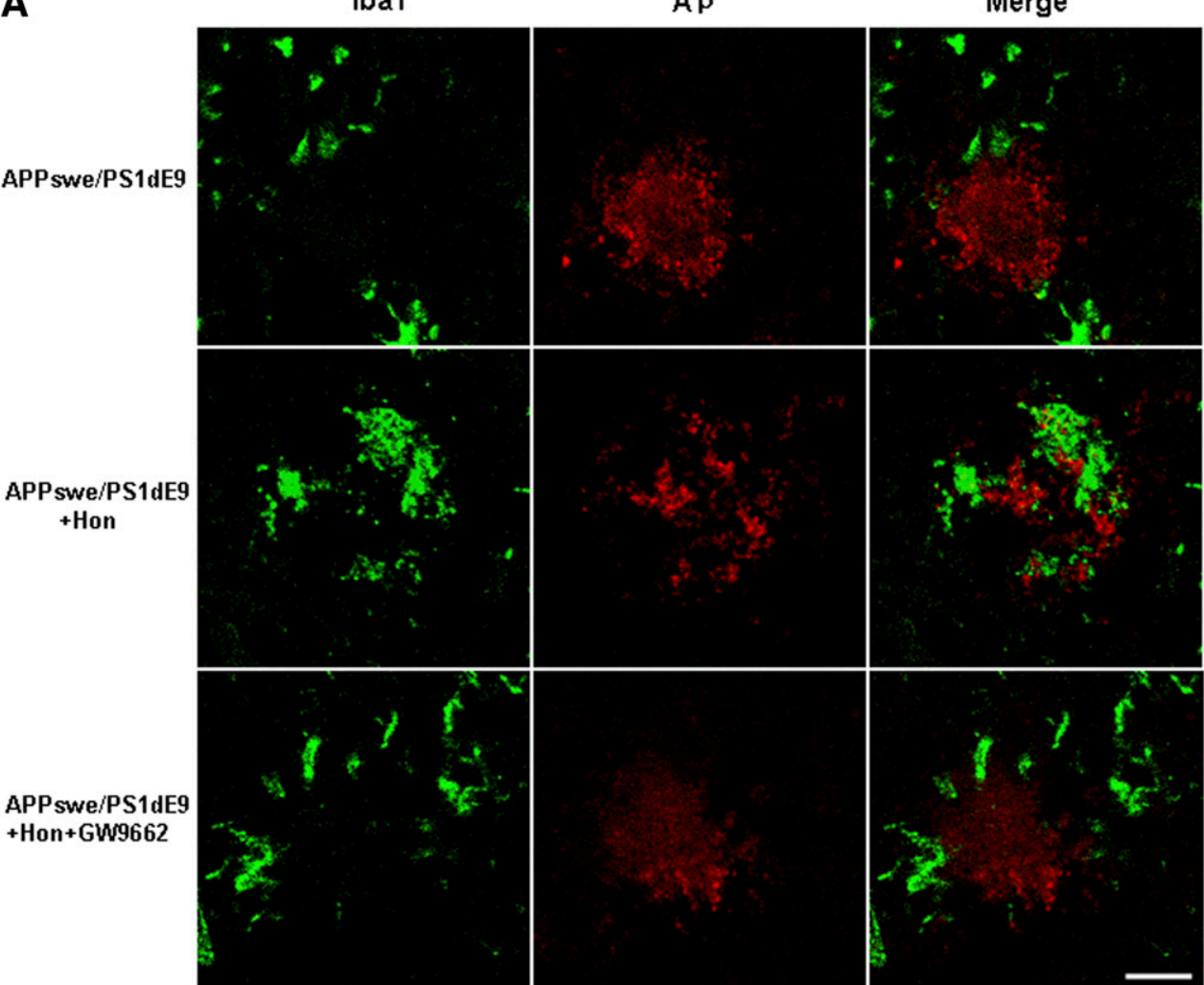

B
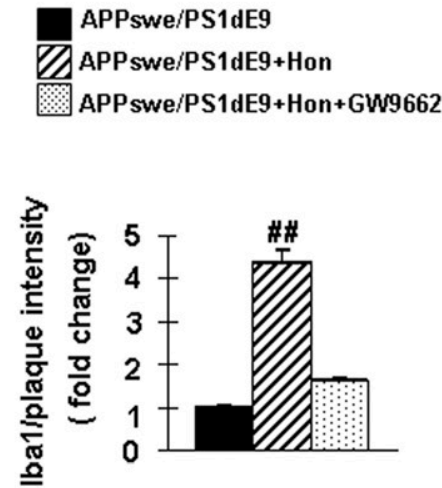

Fig. 5. Honokiol (Hon) treatment increased microglia around the plaque and enhanced A $\beta$ phagocytosis in APPswe/PS1dE9 transgenic mice. (A) Representative images of microglia and $\mathrm{A} \beta$ plaques in the cortex stained with anti-Iba1 (green) and anti-A $\beta$ (red) immunofluorescence (scale bar, $10 \mu \mathrm{m}$ ). Significantly increased Iba1 immunoreactivity around the plaque was found in APPswe/PS1dE9 + Hon mice compared with APPswe/PS1dE9 mice. However, the positive effects of honokiol on $\mathrm{A} \beta$ phagocytosis were reversed by coadministration of GW9662. (B) Ratio of fluorescence intensity of microglial marker Iba1 (green) to amyloid plaque (red). All of the data are presented as mean \pm S.E.M. ( $n=4$ per group) and were analyzed by one-way analysis of variance followed by a Bonferroni post-hoc test for multiple comparisons. ${ }^{\# \#} P<0.01$ vs. APPswe/PS1dE9 mice.

$(P<0.01)$ and $65.5 \%$ in the cortex $(P<0.01)$ (Fig. 3C), and lowered the insoluble $\mathrm{A} \beta 1-42$ levels by $52.5 \%$ in the hippocampus $(P<0.01)$ and $61.8 \%$ in the cortex $(P<0.01)$ (Fig. 3D). Neither soluble $A \beta 1-40$ levels (Fig. 3A) nor insoluble $A \beta 1-40$ levels (Fig. 3B) in the hippocampus and cortex were changed by honokiol treatment. However, intraperitoneal injection of GW9662 almost abolished the effects of honokiol on $\mathrm{A} \beta$ generation and deposition.

Honokiol Suppresses Neuroinflammatory Response and Enhances A $\beta$ Clearance in APPswe/PS1dE9 Mice. The inflammatory response was manifested by an elevation in the levels of TNF- $\alpha$, IL- $1 \beta$, and IL- 6 in the brain of APPswe/PS1dE9 mice. Moreover, glial activation was observed with increased Iba1 and GFAP immunoreactivity as well as the expression of proteins in the hippocampus of APPswe/PS1dE9 mice. Honokiol treatment significantly inhibited neuroinflammatory response, as indicated by decreased levels of TNF- $\alpha$ by $54.7 \%(P<0.01)$, IL- $1 \beta$ by $49.5 \%(P<0.01)$, IL-6 by $51.4 \%(P<0.01)$, reduced number of Iba1 and GFAP positive cells, and lowered expression of proteins compared with those in the APPswe/PS1dE9 mice. Mice treated with only honokiol showed similar neuroinflammatory response as WT mice (Fig. 4). Interestingly, we observed that the microglia around the amyloid plaques was increased in the cortex of honokioltreated APPswe/PS1dE9 mice, suggesting the enhanced $\mathrm{A} \beta$ phagocytosis by microglia (Fig. 5). Addition of GW9662 treatment blocked the inhibitory effects of honokiol on neuroinflammatory response.

Honokiol Improves PPAR $\gamma$ Function and Downregulates BACE1 Activity and Expression in APPswe/PS1dE9 Mice. The above data indicated that PPAR $\gamma$ was associated with the antiamyloid and anti-inflammatory effects of honokiol in APPswe/PS1dE9 mice. Both the expression and activity of -PPAR $\gamma$ were significantly decreased in the hippocampus of APPswe/PS1dE9 mice compared with the WT mice. Honokiol treatment significantly increased PPAR $\gamma$ protein expression (Fig. 6A) as well as PPAR $\gamma$ transcriptional activity (Fig. 6B). Recent studies showed that honokiol could directly bind to PPAR $\gamma$ and act as a PPAR $\gamma$ agonist, which may explain the effects of honokiol on PPAR $\gamma$ functional improvement.

PPAR $\gamma$ suppresses BACE1 activity and expression by binding to the BACE1 gene promoter and subsequently reduces $\mathrm{A} \beta$ generation. To determine whether honokiol affected the BACE1 in APPswe/PS1dE9 mice, we examined the expression and activity of BACE1 in the hippocampus in different groups. The results demonstrated that the expression and activity of BACE1 were elevated in APPswe/PS1dE9 mice compared with those in the WT group and were significantly decreased in honokiol-treated mice (Fig. 6, C and D). Furthermore, GW9662 treatment suppressed the effects of honokiol on the expression and activity of BACE1, suggesting a PPAR $\gamma$-dependent mechanism. No significant difference was observed in the expression and transcriptional 

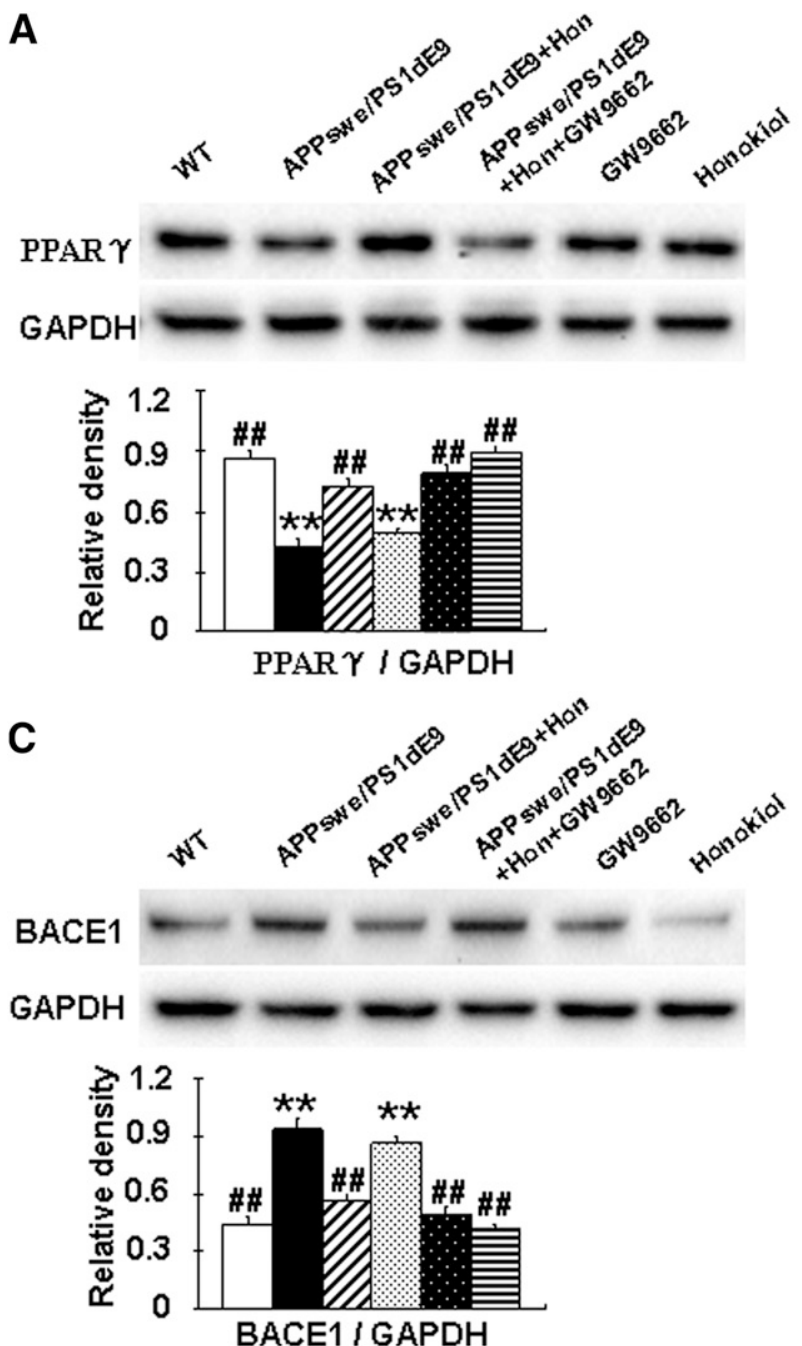

B

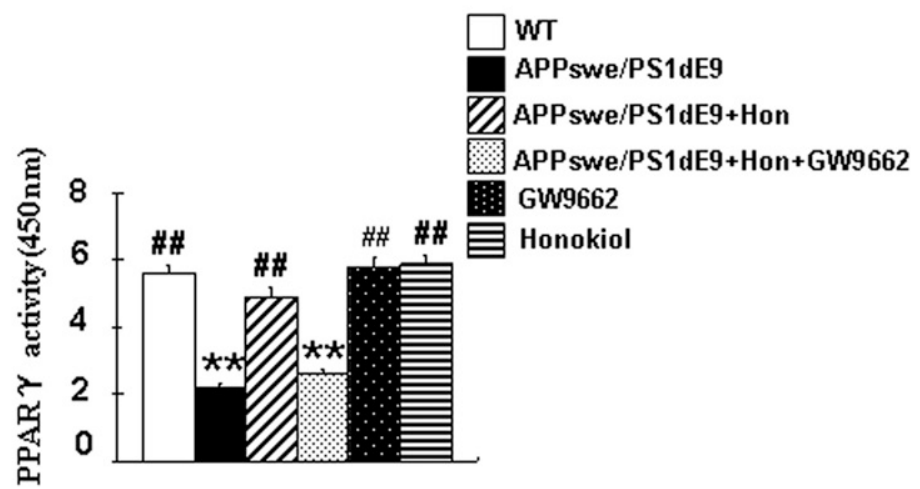

D

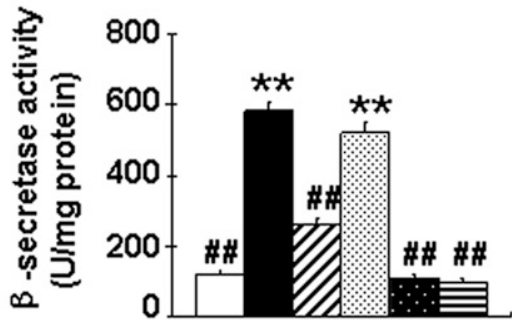

Fig. 6. Honokiol (Hon) improved PPAR $\gamma$ function and decreased BACE1 activity and expression in APPswe/PS1dE9 mice. (A) Western blot of PPAR $\gamma$ expression in the hippocampus. (B) PPAR $\gamma$ transcriptional activity. Honokiol treatment significantly increased PPAR $\gamma$ expression and restored the activity of PPAR $\gamma$ in the brains of APPswe/PS1dE9 mice. However, the protective effects of honokiol on PPAR $\gamma$ function were blocked by coadministration of GW9662. (C) Western blot of BACE1 expression in the hippocampus. (D) $\beta$-secretase activity assay. Honokiol treatment significantly suppressed BACE1 activity and expression in the brains of APPswe/PS1dE9 mice. However, the effects of honokiol on BACE1 activity and expression were also blocked by coadministration of GW9662. All of the data are presented as mean \pm S.E.M. $(n=3$ per group $)$ and were analyzed by one-way

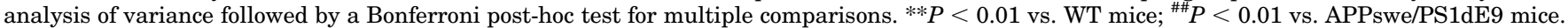

activity of PPAR $\gamma$ or in the expression and activity of BACE1 between mice treated with only honokiol and WT mice.

\section{Discussion}

This study demonstrated that honokiol administration ameliorated spatial learning and memory impairment, reduced plaque burden and $\mathrm{A} \beta$ levels, and suppressed glial activation and the production of neuroinflammatory cytokines in the APPswe/PS1dE9 transgenic mouse model of AD. Furthermore, honokiol treatment improved PPAR $\gamma$ function and downregulated the expression and activity of BACE1. Importantly, the PPAR $\gamma$ inhibitor could block the beneficial effects of honokiol on biochemistry, pathologic changes, and cognitive function in mice.

Although the exact $\mathrm{AD}$ pathogenesis and underlying mechanism remain elusive, the accumulation of $\mathrm{A} \beta$ peptides in the brain is considered to be a central event in AD development (Walsh and Selkoe, 2004; Selkoe and Hardy, 2016). We analyzed neuropathological changes after honokiol treatment and observed the decreased intensity of thioflavin-S-positive staining and A $\beta 1-42$ levels in the brains of honokiol-treated APPswe/PS1dE9 mice. There are two major isoforms of $\mathrm{A} \beta$ peptides: $\mathrm{A} \beta 1-40$ and $\mathrm{A} \beta 1-42$. $\mathrm{A} \beta 1-40$ accumulates in the $\mathrm{AD}$ brain, but the extent of $\mathrm{A} \beta 1-40$ accumulation relative to $\mathrm{A} \beta 1-42$ is highly variable. It has been shown that $\mathrm{A} \beta 1-40$ can promote $\mathrm{A} \beta 42$ aggregation in a concentration-dependent manner (Tran et al., 2017). High A $\beta 1-40$ monomeric/A $\beta 1-42$ fibrillar ratios ( $\geq 10$ ) accelerate $\mathrm{A} \beta$ fibril formation and the accumulation of fibrillar aggregates in the brain. In contrast, subphysiologic $\mathrm{A} \beta 1-40$ monomeric/A $\beta 1-42$ monomeric ratios $(0.5-2)$ facilitate the persistence and accumulation of soluble $\mathrm{A} \beta$ aggregates (Jan et al., 2008). In the present study, the ratio of $\mathrm{A} \beta 1-40 / \mathrm{A} \beta 1-42$ observed was up to 10 , which may promote A $\beta 1-42$ fibril formation. The aggregation of $\mathrm{A} \beta 1-42$ into small oligomers and fibrillar plaques is considered as the central initiator in the pathogenesis of $\mathrm{AD}$ (Lambert et al., 1998; Selkoe, 2011). Therefore, the lowered A $\beta 1-42$ level after 
honokiol treatment may explain its attenuating effects on hippocampus-dependent behavioral deficits.

Neuroinflammation plays a crucial role in the development of neurodegenerative diseases, including $\mathrm{AD}$ (Minter et al., 2016). Glial cells act as neuronal supportive cells and maintain the health of the neurons. However, glial overactivation in response to $\mathrm{A} \beta$ can produce proinflammatory mediators and neurotoxic factors that cause neuronal dysfunction and neurodegeneration, ultimately creating a vicious cycle (Skaper, 2007). Overactivation of microglia and astrocytes and higher levels of proinflammatory cytokines, such as TNF- $\alpha$, IL- $1 \beta$, and IL-6, have been found in the brains of $\mathrm{AD}$ patients (Angelopoulos et al., 2008; Forlenza et al., 2009) and AD animal models (Morales et al., 2010). Consistent with this, our results indicated augmented neuroinflammation, as evidenced by increased proinflammatory levels, and Iba1 and GFAP immunoreactivity as well as the expression of proteins in the brains of APPswe/PS1dE9 mice. Honokiol treatment significantly suppressed the neuroinflammation response.

The important role of the nuclear receptor PPAR $\gamma$ agonists in neuroprotection has been extensively studied in neurodegenerative disorders, including $\mathrm{AD}$ (Sodhi et al., 2011; Mandrekar-Colucci et al., 2012; Heneka et al., 2015). Compelling evidence has shown that PPAR $\gamma$ agonists effectively improve cognitive impairment in $\mathrm{AD}$ patients (Cheng et al., 2016) and AD mouse models (Skerrett et al., 2015). PPAR $\gamma$ is a ligand-dependent transcription factor that regulates the transcription of target genes. In recent years, the modulatory role of PPAR $\gamma$ in the transcriptional activity of BACE1 (Chen et al., 2009; Katsouri et al., 2011), a crucial enzyme in amyloid precursor protein processing and $\mathrm{A} \beta$ production, has been well documented. Previous studies have indicated that PPAR $\gamma$ downregulates BACE1 transcription and exerts effects on $\mathrm{A} \beta$ generation through directly binding to the promoter region of BACE1 (Chen et al., 2009). Consistent with this, our results showed that honokiol treatment enhanced $\operatorname{PPAR} \gamma$ function and suppressed the expression and activity of BACE1, which in turn led to lowered $\mathrm{A} \beta$ production and subsequent senile plaque formation in the brains of APPswe/PS1dE9 mice. However, the effects of honokiol can be effectively blocked by GW9662, a PPAR $\gamma$ antagonist. Therefore, the present data suggested that honokiol represses BACE1 function and $\mathrm{A} \beta$ production by inducing the activation of $\operatorname{PPAR} \gamma$. Meanwhile, the anti-inflammatory action of PPAR $\gamma$ agonists is assumed to underlie their positive effects on the amelioration of $\mathrm{AD}$ pathophysiology (Bright et al., 2008). One striking finding of our study was the extraordinary ability of honokiol to promote microglia to phagocytose $\mathrm{A} \beta$. Although microglia cells are competent phagocytes, they fail to effectively phagocytose $\mathrm{A} \beta$ and have shown impaired ability to degrade $\mathrm{A} \beta$ in the $\mathrm{AD}$ brain (Southam et al., 2016). PPAR $\gamma$ activation has been reported to provoke the conversion of microglia from M1 inflammatory (classic) to M2 anti-inflammatory (alternative) phenotypes and promote $\mathrm{A} \beta$ clearance, resulting in cognitive improvement in the $\mathrm{AD}$ brain (Mandrekar-Colucci et al., 2012; Yamanaka et al., 2012). Thus, in our study, honokiol modulated $\mathrm{A} \beta$ metabolism by inhibiting $\mathrm{A} \beta$ generation via downregulating BACE1 activity and enhancing $\mathrm{A} \beta$ clearance by microglia in APPswe/PS1dE9 mice.

Honokiol remarkably improved spatial learning and memory impairment in an APPswe/PS1dE9 transgenic mouse model of $\mathrm{AD}$. The cognitive-enhancing mechanism of honokiol is likely attributable to decreased $\mathrm{A} \beta$ generation and enhanced $\mathrm{A} \beta$ clearance, along with suppressed neuroinflammation; these effects appear to be due to the activation of $\operatorname{PPAR} \gamma$. Therefore, honokiol might be a potential natural compound candidate against $\mathrm{AD}$.

\section{Authorship Contributions}

Participated in research design: D. Wang, Dong, C. Wang.

Conducted experiments: D. Wang, Dong, C. Wang.

Performed data analysis: D. Wang, Dong.

Wrote or Contributed to the writing of the manuscript: D. Wang, C. Wang.

\section{References}

Angelopoulos P, Agouridaki H, Vaiopoulos H, Siskou E, Doutsou K, Costa V, and Baloyiannis SI (2008) Cytokines in Alzheimer's disease and vascular dementia. Int $J$ Neurosci 118:1659-1672.

Atanasov AG, Wang JN, Gu SP, Bu J, Kramer MP, Baumgartner L, Fakhrudin N, Ladurner A, Malainer C, Vuorinen A, et al. (2013) Honokiol: a non-adipogenic PPAR $\gamma$ agonist from nature. Biochim Biophys Acta 1830:4813-4819.

Bright JJ, Kanakasabai S, Chearwae W, and Chakraborty S (2008) PPAR regulation of inflammatory signaling in CNS diseases. PPAR Res 2008:658520.

Chen Y, Zhou K, Wang R, Liu Y, Kwak YD, Ma T, Thompson RC, Zhao Y, Smith L, Gasparini L, et al. (2009) Antidiabetic drug metformin (GlucophageR) increases biogenesis of Alzheimer's amyloid peptides via up-regulating BACE1 transcription. Proc Natl Acad Sci USA 106:3907-3912.

Cheng H, Shang Y, Jiang L, Shi TL, and Wang L (2016) The peroxisome proliferators activated receptor-gamma agonists as therapeutics for the treatment of Alzheimer's disease and mild-to-moderate Alzheimer's disease: a meta-analysis. Int $J$ Neurosci 126:299-307.

de la Monte SM and Wands JR (2008) Alzheimer's disease is type 3 diabetesevidence reviewed. J Diabetes Sci Technol 2:1101-1113.

Escribano L, Simón AM, Gimeno E, Cuadrado-Tejedor M, López de Maturana R, García-Osta A, Ricobaraza A, Pérez-Mediavilla A, Del Río J, and Frechilla D (2010) Rosiglitazone rescues memory impairment in Alzheimer's transgenic mice: mechanisms involving a reduced amyloid and tau pathology. Neuropsychopharmacology 35:1593-1604

Forlenza OV, Diniz BS, Talib LL, Mendonça VA, Ojopi EB, Gattaz WF, and Teixeira AL (2009) Increased serum IL- $1 \beta$ level in Alzheimer's disease and mild cognitive impairment. Dement Geriatr Cogn Disord 28:507-512.

Handattu SP, Garber DW, Monroe CE, van Groen T, Kadish I, Nayyar G, Cao D Palgunachari MN, Li L, and Anantharamaiah GM (2009) Oral apolipoprotein A-I mimetic peptide improves cognitive function and reduces amyloid burden in a mouse model of Alzheimer's disease. Neurobiol Dis 34:525-534.

Hardy J and Selkoe DJ (2002) The amyloid hypothesis of Alzheimer's disease: progress and problems on the road to therapeutics. Science 297:353-356.

Heneka MT, Fink A, and Doblhammer G (2015) Effect of pioglitazone medication on the incidence of dementia. Ann Neurol 78:284-294.

Jan A, Gokce O, Luthi-Carter R, and Lashuel HA (2008) The ratio of monomeric to aggregated forms of $\mathrm{A} \beta 40$ and $\mathrm{A} \beta 42$ is an important determinant of amyloid- $\beta$ aggregation, fibrillogenesis, and toxicity. J Biol Chem 283:28176-28189.

Katsouri L, Parr C, Bogdanovic N, Willem M, and Sastre M (2011) PPAR co-activator- $1 \alpha$ (PGC-1 $\alpha$ ) reduces amyloid- $\beta$ generation through a PPAR $\gamma$-dependent mechanism. J Alzheimers Dis 25:151-162.

Lambert MP, Barlow AK, Chromy BA, Edwards C, Freed R, Liosatos M, Morgan TE, Rozovsky I, Trommer B, Viola KL, et al. (1998) Diffusible, nonfibrillar ligands derived from $\mathrm{A} \beta_{1-42}$ are potent central nervous system neurotoxins. Proc Natl Acad Sci USA 95:6448-6453.

Lee YJ, Choi DY, Yun YP, Han SB, Kim HM, Lee K, Choi SH, Yang MP, Jeon HS, Jeong JH, et al. (2013) Ethanol extract of Magnolia officinalis prevents lipopolysaccharide-induced memory deficiency via its antineuroinflammatory and antiamyloidogenic effects. Phytother Res 27:438-447.

Li SQ, Wang DM, Shu YJ, Wan XD, Xu ZS, and Li EZ (2013) Proper heat shock pretreatment reduces acute liver injury induced by carbon tetrachloride and accelerates liver repair in mice. J Toxicol Pathol 26:365-373.

Liu Y, Zhang Z, Qin Y, Wu H, Lv Q, Chen X, and Deng W (2013) A new method for Schwann-like cell differentiation of adipose derived stem cells. Neurosci Lett 551:79-83.

Maher PA and Schubert DR (2009) Metabolic links between diabetes and Alzheimer's disease. Expert Rev Neurother 9:617-630.

Mandrekar-Colucci S, Karlo JC, and Landreth GE (2012) Mechanisms underlying the rapid peroxisome proliferator-activated receptor- $\gamma$-mediated amyloid clearance and reversal of cognitive deficits in a murine model of Alzheimer's disease. $J$ Neurosci 32:10117-10128.

Matsui N, Takahashi K, Takeichi M, Kuroshita T, Noguchi K, Yamazaki K, Tagashira H, Tsutsui K, Okada H, Kido Y, et al. (2009) Magnolol and honokiol prevent learning and memory impairment and cholinergic deficit in SAMP8 mice. Brain Res 1305:108-117.

Minter MR, Taylor JM, and Crack PJ (2016) The contribution of neuroinflammation to amyloid toxicity in Alzheimer's disease. J Neurochem 136:457-474.

Morales I, Farías G, and Maccioni RB (2010) Neuroimmunomodulation in the pathogenesis of Alzheimer's disease. Neuroimmunomodulation 17:202-204.

Pérez MJ and Quintanilla RA (2015) Therapeutic actions of the thiazolidinediones in Alzheimer's disease. PPAR Res 2015:957248.

Searcy JL, Phelps JT, Pancani T, Kadish I, Popovic J, Anderson KL, Beckett TL, Murphy MP, Chen KC, Blalock EM, et al. (2012) Long-term pioglitazone treatment improves learning and attenuates pathological markers in a mouse model of Alzheimer's disease. J Alzheimers Dis 30:943-961. 
Selkoe DJ (2011) Resolving controversies on the path to Alzheimer's therapeutics. Nat Med 17:1060-1065.

Selkoe DJ and Hardy J (2016) The amyloid hypothesis of Alzheimer's disease at 25 years. EMBO Mol Med 8:595-608.

Skaper SD (2007) The brain as a target for inflammatory processes and neuroprotective strategies. Ann N Y Acad Sci 1122:23-34.

Skerrett R, Pellegrino MP, Casali BT, Taraboanta L, and Landreth GE (2015) Combined liver X receptor/peroxisome proliferator-activated receptor $\gamma$ agonist treatment reduces amyloid $\beta$ levels and improves behavior in amyloid precursor protein/presenilin 1 mice. J Biol Chem 290:21591-21602.

Sodhi RK, Singh N, and Jaggi AS (2011) Neuroprotective mechanisms of peroxisome proliferator-activated receptor agonists in Alzheimer's disease. Naunyn Schmiedebergs Arch Pharmacol 384:115-124.

Southam KA, Vincent AJ, and Small DH (2016) Do microglia default on network maintenance in Alzheimer's disease? J Alzheimers Dis 51:657-669.

Steel K (2010) Alzheimer's disease. N Engl J Med 362:1844-1845.

Talbot K, Wang HY, Kazi H, Han LY, Bakshi KP, Stucky A, Fuino RL, Kawaguchi KR, Samoyedny AJ, Wilson RS, et al. (2012) Demonstrated brain insulin resistance in Alzheimer's disease patients is associated with IGF-1 resistance, IRS-1 dysregulation, and cognitive decline. J Clin Invest 122:1316-1338.

Tran J, Chang D, Hsu F, Wang H, and Guo Z (2017) Cross-seeding between A $\beta 40$ and A $\beta 42$ in Alzheimer's disease. FEBS Lett 591:177-185.

Walsh DM and Selkoe DJ (2004) Deciphering the molecular basis of memory failure in Alzheimer's disease. Neuron 44:181-193.
Wang H, Liao Z, Sun X, Shi Q, Huo G, Xie Y, Tang X, Zhi X, and Tang Z (2014) Intravenous administration of Honokiol provides neuroprotection and improves functional recovery after traumatic brain injury through cell cycle inhibition. Neuropharmacology 86:9-21.

Wang X, Duan X, Yang G, Zhang X, Deng L, Zheng H, Deng C, Wen J, Wang N, Peng C, et al. (2011) Honokiol crosses BBB and BCSFB, and inhibits brain tumor growth in rat 9L intracerebral gliosarcoma model and human U251 xenograft glioma model. PLoS One 6:e18490.

Yamanaka M, Ishikawa T, Griep A, Axt D, Kummer MP, and Heneka MT (2012) PPAR $\gamma / \mathrm{RXR} \alpha$-induced and CD36-mediated microglial amyloid- $\beta$ phagocytosis results in cognitive improvement in amyloid precursor protein/presenilin 1 mice. $J$ Neurosci 32:17321-17331.

Yuan SM, Gao K, Wang DM, Quan XZ, Liu JN, Ma CM, Qin C, and Zhang LF (2011) Evodiamine improves congnitive abilities in SAMP8 and APP(swe)/ PS1 $(\Delta \mathrm{E} 9)$ transgenic mouse models of Alzheimer's disease. Acta Pharmacol Sin 32:295-302.

Address correspondence to: Dongmei Wang, Department of Pathogen Biology, Medical College, Henan University of Science and Technology, No. 263, Kaiyuan Avenue, Luolong District, Luoyang 471023, China. E-mail: wdmzgadyx@hotmail.com 